\title{
Injection liquide dans les foyers de turboréacteurs : problèmes industriels et modélisation
}

\author{
- Liquid injection in turboreactors combustors : \\ industrial problems and modelling -
}

par C. Dejeu, C. Baudoin

Snecma Villaroche

This article presents a state of the art of the different technologies of fuel injectors used by Snecma in aeronautical engines. Traditionnal techniques of injection as well as concepts are described. The major role of the injector on the global performances of the combustor is pointed out. The experimental set up for the caracterisation of injection systems (granulometry, measurement of the spray angle, etc.) are presented. Finally, the authors will mention the numerical modelling developed for the simulation of the complex problem of the two-phase reactive flows in aeronautical combustors.

\section{I 口 INTRODUCTION}

Dans les turbomachines aéronautiques, les chambres de combustion sont placées entre le compresseur et la turbine pour le foyer principal et en aval de la turbine pour le foyer de réchauffe lorsqu'il existe. C'est au niveau de ces composants que l'énergie est apportée au fluide par la combustion d'un hydrocarbure. Le carburant est pulvérisé dans la chambre de combustion sous la forme d'un brouillard de gouttelettes liquides qui seront ensuite transportées, évaporées par l'écoulement turbulent. Le choix et le dimensionnement d'un système d'injection constitue un des points cruciaux dans le dimensionnement d'un foyer aéronautique. En effet, une grande partie des performances d'une chambre de combustion est déterminée par la qualité du mélange local air/carburant (carte de richesse). Ainsi, le rendement de combustion, le niveau de formation de polluants, la stabilité du foyer, le niveau local de température sont, entre autres, des fonctions directes du système d'injection.

La mise au point et l'amélioration des foyers aéronautiques et de leurs injecteurs fut dans un premier temps le résultat de la conjugaison de calculs élémentaires et de nombreux essais au banc. L'empirisme (corrélations diverses) et l'expérience du motoriste étaient alors la règle essentielle pour dimensionner. Depuis les années 80 , la modélisation et la simulation numérique ont pris un essor très important, pour devenir à l'heure actuelle des éléments majeurs de la recherche, de l'étude, de la conception et du développement des foyers. Les études expérimentales demeurent néanmoins plus que jamais indispensables pour valider les choix technologiques issus d'une étude numérique détaillée. Elles sont également largement mises en œuvre avec des moyens de mesures de plus en plus sophistiqués dans un but de validation des codes de calcul.

\section{II $\square$ LE FONCTIONNEMENT DES FOYERS DE TURBORÉACTEUR (CHAMBRE PRINCIPALE)}

Dans un turboréacteur, la chambre de combustion principale sert à augmenter l'enthalpie et la température de l'air issu du compresseur par combustion du carburant injecté sous la forme d'un nuage de gouttelettes liquides.

Un foyer d'architecture classique est annulaire. Il est alimenté en carburant par plusieurs injecteurs répartis régulièrement dans le fond de chambre.

Le dimensionnement d'un foyer de turboréacteur résulte d'un compromis entre les différentes performances à des conditions de fonctionnement extrêmes : ralenti et plein gaz (décollage). Cela est la différence majeure entre une turbine à gaz terrestre qui fonctionne uniquement à pression atmosphérique et un turboréacteur qui fonctionne dans une plage de fonctionnement étendue : les pressions varient de 0,2 à 40 bars et les températures d'entrée chambre varient de 220 à $900 \mathrm{~K}[1]$.

Il apparaît que le système d'injection est l'élément essentiel d'un foyer de turboréacteur puisqu'il influence directement la quasi-totalité des performances du foyer. Le système d'injection est constitué chez Snecma de l'injecteur de carburant proprement dit et d'un système de vrilles associé permettant l'admission de l'air dans le fond de la chambre avec giration (ou swirl). C'est le système d'injection et non seulement l'injecteur qui va déterminer la granulométrie du brouillard de carburant et la distribution spatiale des gouttes, qui va contribuer à établir la carte de richesse à l'intérieur du 


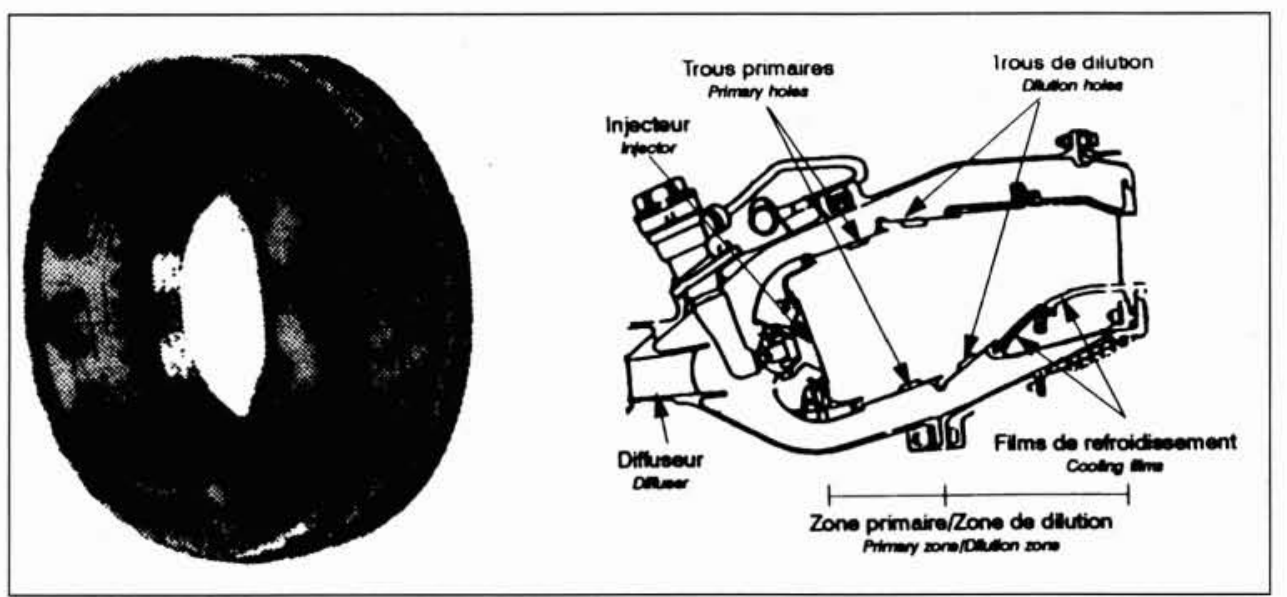

1. Foyer conventionnel.

foyer. Ces deux caractéristiques du système d'injection résultent de l'étroit couplage entre l'injecteur de carburant et les tourbillons d'air générés par les vrilles. Ainsi, la maîtrise de la distribution de richesse par une optimisation du système d'injection est une phase critique lors du dimensionnement d'un foyer.

\section{III — LES DIFFÉRENTES TECHNOLOGIES D'INJECTION}

\section{- 3.1 Les architectures traditionnelles}

La mise au point de systèmes d'injection de foyers aéronautiques fut pendant de nombreuses années basée sur un savoir-faire issu de calculs élémentaires et de nombreux essais au banc. Jusqu'à la fin des années 1980, le dimensionnement était uniquement basé sur une approche empirique, le savoir-faire du constructeur et sur les progrès de la technologie (miniaturisation du dispositif, maîtrise des procédés de fabrication). De manière globale, on recherche lors de la mise au point d'un système d'injection à répartir le carburant liquide dans la chambre de combustion pour obtenir un brouillard (ou spray en terminologie anglo-saxone) le plus homogène possible, de façon à limiter les hétérogénéités de richesse. On cherche également, en général, à obtenir un brouillard constitué de gouttes les plus petites possibles pour limiter la durée d'évaporation, brûler le plus tôt possible et réduire la longueur du foyer.

Au fil des années, différents types de systèmes d'injection, à la pointe de la technologie, ont été utilisés dans les moteurs Snecma (fig 2) :
- injecteur mécanique : la pulvérisation de carburant est réalisée par une mise en pression de celui-ci avant injection. Ce dispositif est utilisé sur la chambre ATAR qui équipe les Mirage III, IV et F1, la surpression d'injection étant de l'ordre de 30 bars. L'injecteur comporte un double circuit de façon à assurer une pulvérisation correcte à la fois au régime ralenti et au plein gaz.

- cannes à prévaporisation où l'on réalise un prémélange air/carburant en vaporisant de façon partielle le carburant dans un canal à retour, l'injection du prémélange s'effectuant vers le fond de chambre. Cette technique est employée sur le moteur M53 qui équipe le Mirage 2000 ainsi que sur l'Olympus du Concorde.

— injecteur aérodynamique : la pulvérisation du carburant est ici assurée par la création d'instabilités hydrodynamiques d'une nappe circulaire de carburant située entre deux nappes d'air corotatives. La nappe de carburant est fragmentée en ligaments qui se brisent ensuite pour générer le spray de carburant. Ce type de système est utilisé sur le moteur M88 du Rafale.

On rencontre différents problèmes lors de la mise au point des systèmes d'injection : le carburant liquide peut ruisseler sur les parois chaudes au voisinage de l'injecteur et pyrolyser pour former du coke, qui peut boucher partiellement les orifices de sortie du carburant. L'échauffement de l'injecteur par le rayonnement de la flamme peut également être préjudiciable pour la pulvérisation. En effet, le carburant liquide peut atteindre son point de vaporisation, générer des bulles de vapeur bloquant la sortie du liquide (phénomène de vapor lock). Par conséquent, le refroidissement de l'injecteur doit être assuré avec beaucoup de soin.

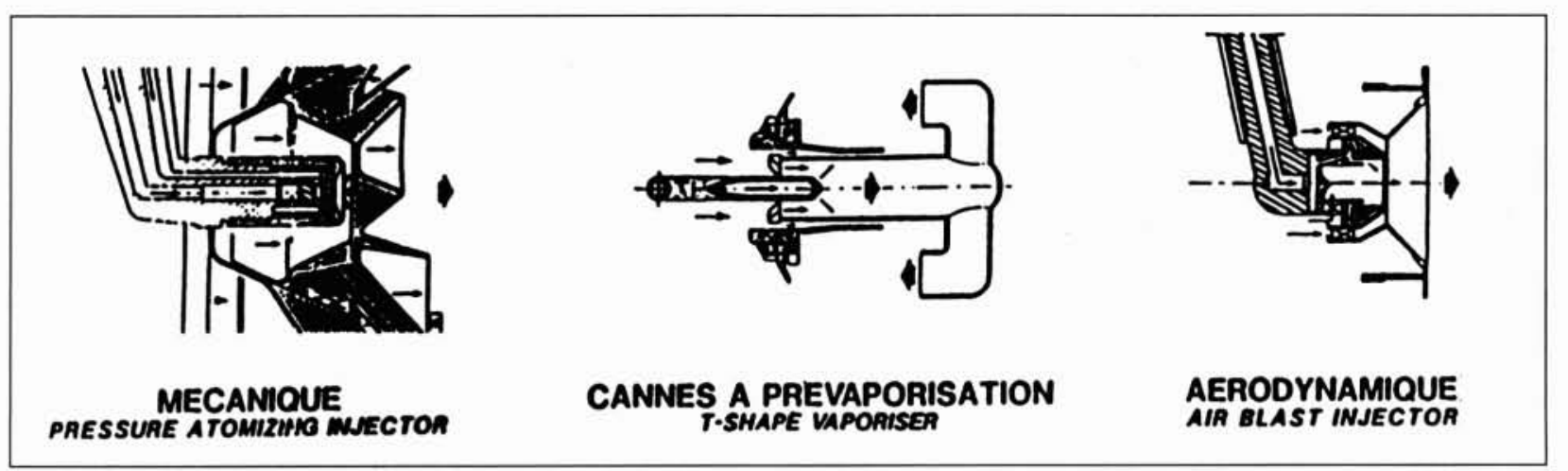

2. Technologies d'injection. 


\section{- 3.2 Les nouveaux concepts}

Nous avons vu précédemment que le système d'injection conditionne la carte de richesse tridimensionnelle à l'intérieur du foyer et par là même la formation de polluants tels que les oxydes d'azote (NOx), le monoxyde de carbone et les suies. Les réglementations ou les exigences de certains clients concernant la pollution ont tendance à devenir de plus en plus sévères, principalement pour les oxydes d'azote. Pour faire face à ces nouvelles préoccupations en matière d'environnement, Snecma a engagé des nouvelles voies de recherche technologique sur l'architecture des foyers et sur les systèmes d'injection.

Une des voies prometteuses pour réduire les émissions d'oxydes d'azote est le foyer à prémélange LPP (Lean Premixed Prevaporized). L'objectif visé est une réduction des NOx jusqu'à $80 \%$ par rapport à une architecture conventionnelle. La figure 3 montre un exemple de ce type de foyer. Il s'agit d'assurer une prévaporisation et un prémélange homogène air/carburant dans un tube de prémélange avant d'opérer la combustion. Le rôle de l'injecteur est ici critique car il doit fournir une granulométrie du brouillard caractérisée par un faible diamètre moyen de Sauter (SMD) afin de minimiser le délai d'évaporation. En effet, l'inconvénient majeur de ce concept est le risque d'auto inflammation du prémélange en amont du foyer et la remontée de flamme (flash back) jusqu'à l'injecteur.

Une autre voie en cours de développement pour réduire les niveaux de pollution en NOx concerne la mise au point de systèmes d'injection à géométrie variable. Il s'agit de moduler le débit d'air passant en fond de chambre pour satisfaire au compromis de dimensionnement entre ralenti et plein gaz. L'injecteur choisi pour les configurations à géométries variables est de type aéromécanique. L'injection est effectuée par un injecteur mécanique puis relayée par une pulvérisation secondaire engendrée par le système de vrilles associé permettant l'admission de l'air avec giration. (fig 4).

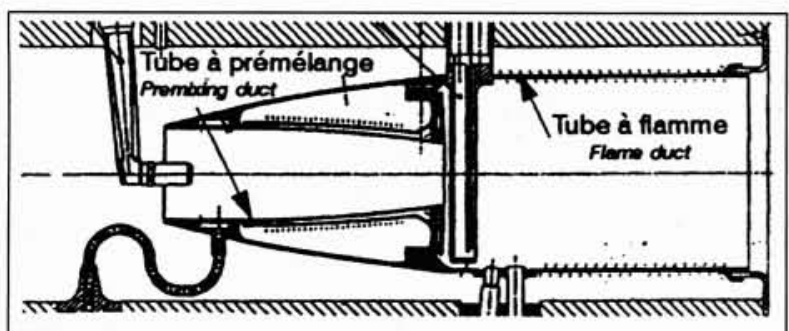

3. Foyer LPP.

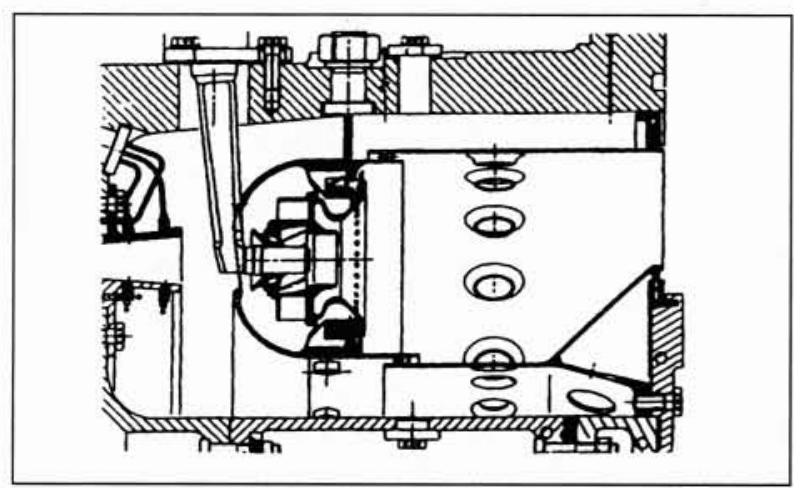

4. Foyer à géométrie variable.

\section{MISE AU POINT EXPÉRIMENTALE}

Le développement de nouveaux systèmes d'injection passe après la phase de conception et de définition par une série d'essais au banc, en général à pression et température ambiante pour des raisons de facilité de réalisation.

Deux types d'essais sont couramment effectués à la Snecma Villaroche :

* les essais de pluviométrie,

* les essais de granulométrie MALVERN.

Des caractérisations plus fines de l'injecteur (PDPA) sont de plus réalisées à l'ONERA/CERT

\subsection{Essais de pluviométrie}

Ces essais visent à caractériser la répartition spatiale du carburant liquide, indépendamment de la granulométrie du spray. On dispose donc à une certaine distance de l'injecteur une série d'éprouvettes qui vont recueillir le carburant situé dans l'angle solide de celles-ci. On observe expérimentalement que le débit de carburant se situe globalement sur un cône creux dont les essais de pluviométrie fournissent l'angle d'ouverture maximum $\mathrm{a}_{90 \%}$ et l'angle d'ouverture minimum $\mathrm{a}_{10 \%}$ où $\mathrm{a}_{\mathrm{x} \%}$ représente l'angle dans lequel on retrouve $\mathrm{x} \% \mathrm{du}$ débit liquide. On vérifie également lors des essais de pluviométrie que la carburation ne présente pas d'hétérogénéités circonférentielles. On trouvera sur la figure 5 une vue du dispositif expérimental.

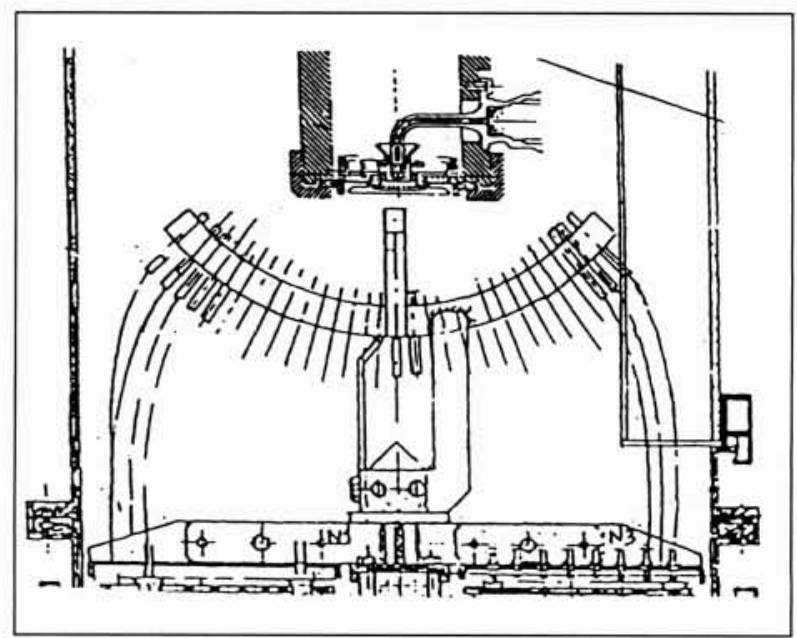

5. Essai de pluviométrie.

\section{- 4.2 Essais de granulométrie MALVERN.}

Ces essais sont basés sur une mesure de tailles de gouttes, résultant de la diffraction de la lumière (diffraction de Fraunhofer) provenant d'un rayon LASER Hélium Néon de longueur d'onde $0,6328 \mu \mathrm{m}$, dont la visée traverse le spray.

En fonction de l'intensité de la lumière diffractée, on tire la distribution en taille des gouttes sur la population présente sur le trajet de visée LASER.

Différents diamètres caractéristiques du spray sont alors calculés, tels que le diamètre moyen de Sauter (ou SMD ou $\mathrm{D}_{32}$ ). Ce diamètre est un paramètre très utile en combustion puisqu'il représente le diamètre d'une goutte dont le rapport volume/surface est égal à celui du spray entier. Ce diamètre permet donc de déduire un temps moyen d'échauffement et d'évaporation pour le spray, paramètres dimensionnants pour 
la longueur du foyer. Le principal défaut de la méthode de mesure par granulométrie Malvern repose sur le fait que seules des informations globales sur l'axe de visée sont accessibles et non pas des données locales.

\section{- 4.3 Essais de granulométrie/vélocimétrie PDPA}

Le granulomètre optique PDPA (Phase Doppler Particle Analyser) est basé sur le principe de l'anémométrie Doppler Laser. Le volume de mesure est constitué par l'intersection de 2 faisceaux laser. Une particule se déplaçant dans ce volume diffuse un rayonnement dans tout l'espace ; la vitesse de la particule est déduite de la fréquence du signal recueilli (effet Doppler), sa taille est déduite de sa phase. Ce type de granulomètre fournit alors une distribution jointe taille/vitesse. Cela représente l'intérêt majeur de cette technique, qui permet en outre d'accéder à des données locales au volume de mesure. Toutefois, cet appareil ne peut pas mesurer les très grosses particules, ainsi que les particules non sphériques qui sont éliminées.

\subsection{Essais en combustion}

Une fois l'injecteur caractérisé en pluviométrie et granulométrie à pression et température ambiante par les techniques mentionnées ci-dessus, il convient de s'assurer du bon comportement global du système d'injection à des conditions de fonctionnement réalistes de la chambre, et en combustion. En particulier, on cherchera à mesurer les concentrrations de diverses espèces $(\mathrm{CO}, \mathrm{NOx}$, fumées, imbrûlés,...) et les limites d'extinction pauvre du foyer. Pour cela, on effectue des essais avec un injecteur seul, monté sur un foyer tubulaire. Ces essais peuvent être effectués à haute pression et haute température, conditions représentatives du fonctionnement d'un foyer.

\section{MODÉLISATION DIPHASIQUE DES SYSTÈMES D'INJECTION}

\section{- 5.1 Hypothèses de la modélisation diphasique}

Dans le but de réduire les essais de mise au point des systèmes d'injection, et de permettre la réalisation d'études paramétriques intensives, Snecma a de plus en plus recours à la modélisation numérique comme véritable outil de recherche, d'étude et de conception.

La modélisation de la phase liquide injectée dans les foyers de turboréacteurs nécessite la prise en compte de phénomènes complexes et variés :
- transfert de masse (évaporation), quantité de mouvement, et d'énergie entre la phase liquide et la phase gazeuse,

- dispersion turbulente du nuage de gouttes injectées,

- interaction entre gouttes (impact, coalescence, modification de la traînée et de l'évaporation),

- interaction entre gouttes et paroi (impact, rebond, ruissellement, ...),

- fragmentation et pulvérisation d'une nappe ou d'un jet liquide (pulvérisation primaire),

- fragmentation d'une goutte en plusieurs gouttes plus petites (pulvérisation secondaire).

Ainsi, on est amené à faire des hypothèses restrictives et simplificatrices pour traiter le problème de la modélisation du brouillard de carburant injecté :

- H1 : la phase liquide est supposée dispersée en un nombre de gouttes important, de sorte que le mouvement de chaque particule soit contrôlé principalement par les forces aérodynamiques et de volume, c'est-à-dire que les forces d'interaction entre gouttes sont négligées. Cette hypothèse très simplificatrice permet de considérer chaque goutte comme isolée de ses voisines.

- H2 : les gouttes sont supposées sphériques, la taille de la particule est inférieure à l'échelle turbulente de Kolmogorov de sorte que l'on suppose que les particules sont entourées d'un écoulement uniforme à l'échelle du maillage aérothermochimique utilisé.

Comme la phase liquide est dispersée, on choisit de simuler le spray par un nombre statistiquement suffisant de particules que l'on étudiera par une méthode lagrangienne de suivi de trajectoires. Cette formulation lagrangienne a l'avantage d'être très précise au niveau du traitement individuel d'une particule mais présente l'inconvénient d'une certaine lourdeur pour l'intégration de l'évolution des gouttes sur leur trajectoire. En outre, pour être suffisamment précise, une méthode lagrangienne nécessite le calcul d'un nombre statistiquement suffisant de trajectoires, typiquement pour nos applications de l'ordre du million, ce qui alourdit sensiblement les temps calcul.

\section{- 5.2 Trajectographie d'une goutte isolée}

D'après les hypothèses décrites précédemment, nous avons à résoudre le problème du calcul de la trajectoire d'une goutte isolée, en prenant en compte l'échauffement et l'évaporation de celle-ci, dans un écoulement gazeux turbulent.

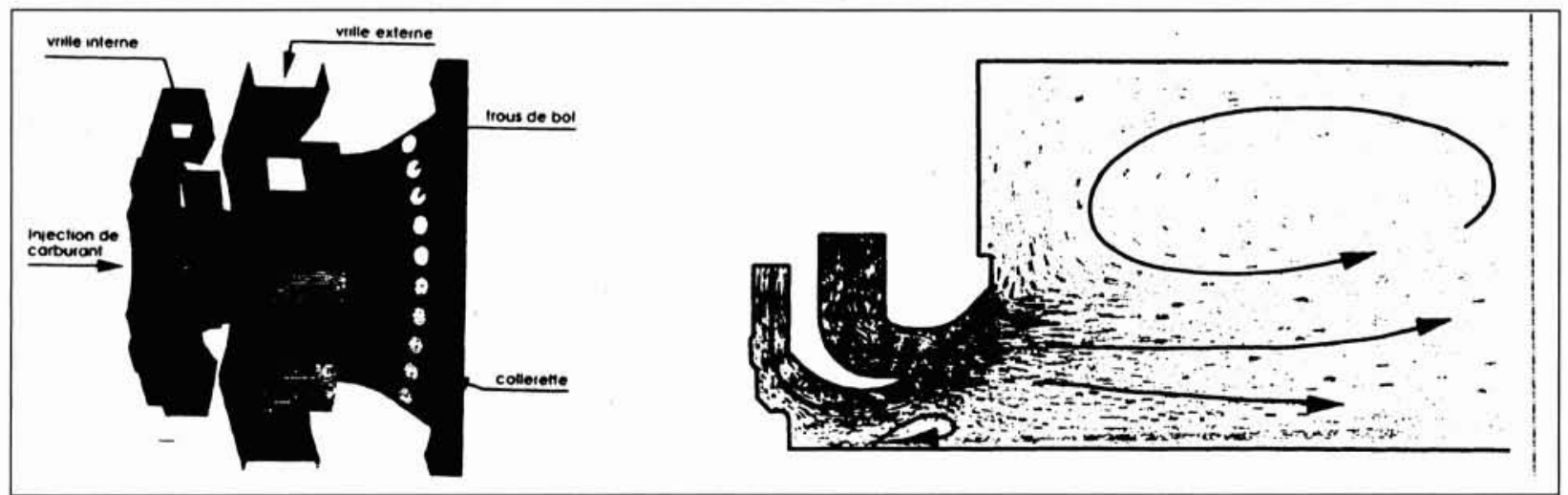

6. Maillage et structure de l'écoulement dans un système d'injection. 


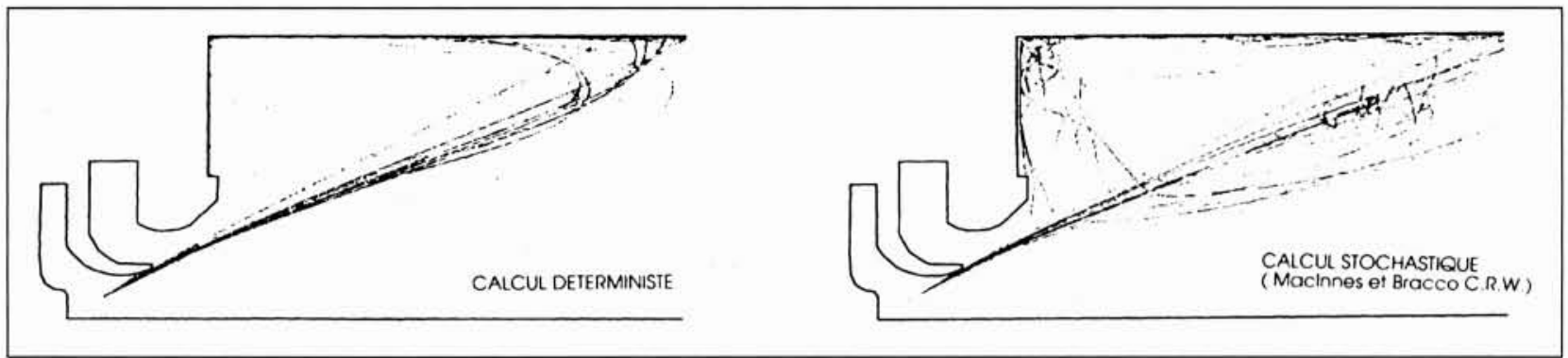

7. Trajectoires de gouttes de carburant en déterministe et en stochastique.

Les caractéristiques aérothermochimiques de l'écoulement gazeux sont calculées classiquement à l'aide des codes de calculs Navier Stokes utilisés par Snecma (DIAMANT code structuré [2] ou NATUR code non structuré [3]). Nous avons donc développé un module diphasique lagrangien utilisable aussi bien sur un maillage structuré que non structuré. En effet, le calcul de la trajectographie des gouttes peut a priori se faire sur un maillage quelconque mais pour des raisons de commodité évidentes, il est préférable d'utiliser le même maillage que celui employé pour le calcul aérothermochimique (fig 6).

L'équation de la trajectoire d'une goutte isolée s'écrit de manière classique en utilisant l'équation fondamentale de la mécanique. Parmi les forces appliquées à la goutte, on peut dénombrer : les forces d'origine aérodynamiques (traînée, portance), la force d'histoire (ou de Basset), la force de masse ajoutée, et les forces de volume (gravité) [4]. Pour nos applications de foyers aéronautiques, où la densité de la phase liquide est nettement supérieure à la densité de l'écoulement porteur gazeux, on peut simplifier l'équation du mouvement de la goutte en ne conservant comme seules forces extérieures que la traînée et les forces de gravité [5].

Le système d'équations définissant la trajectoire peut alors s'écrire:

$$
\frac{d \vec{V}_{p}}{d t}=\frac{\vec{V}_{g}-\vec{V}_{p}}{\tau}+\vec{g} \quad \text { et } \quad \frac{d \vec{X}_{p}}{d t}=\vec{V}_{p}
$$

où l'on a fait apparaître le temps de relaxation $\tau$ qui est le temps caractéristique pour que la goutte atteigne la vitesse du gaz supposée constante. $\tau$ est fonction de la taille de la particule (inertie de la goutte), du coefficient de traînée et du différentiel de vitesse gaz/goutte. [6]

La vitesse $\mathrm{V}_{\mathrm{g}}$ est la vitesse instantanée de l'écoulement porteur gazeux que voit la particule au cours de son mouvement. Pour prendre en compte l'effet de la turbulence de l'air sur la dispersion des gouttes, on utilise un modèle stochastique qui reconstitue la vitesse instantanée du gaz par somme de la vitesse moyenne calculée par le code aérothermochimique et d'une vitesse fluctuante, obtenue par tirage au sort à partir d'une distribution gaussienne de moyenne nulle et d'écart type $(2 / 3 \mathrm{k})^{1 / 2}$ où $\mathrm{k}$ représente l'énergie cinétique de turbulence calculée à partir du modèle $(k, \varepsilon)$.

Le système d'équations (1) est intégré de proche en proche, avec un pas d'intégration en temps variable, qui assure que la goutte se situe toujours sur les faces des éléments du maillage. Le long de la trajectoire, on étudie également l'échauffement et l'évaporation de la goutte concernée par des modèles classiques tels que la loi en D2 ou autres [5] (fig 7). Cela facilite grandement le traitement numérique car on connaît la maille à l'intérieur de laquelle se situe la goutte, à chaque pas d'intégration, ce qui permet de déterminer aisément les termes d'intéraction entre phase liquide et phase gazeuse (taux d'évaporation, énergie échangée pour l'échauffement et l'évaporation des gouttes, échange de quantité de mouvement, ...) dans chaque maille. Ces termes d'interaction entre phases devront être pris en compte lors d'un futur calcul de la phase gazeuse en eulérien, notamment en combustion.

\subsection{Couplage entre phases}

Le calcul diphasique lagrangien tel que nous l'avons décrit permet de transporter un nombre de gouttes représentatif du spray de carburant et d'étudier l'évolution des gouttes sur leur trajectoire physique. Il en résulte des termes d'échange entre phases par l'intermédiaire des transferts de masse (évaporation), de quantité de mouvement (force de traînée) et d'énergie (échauffement et évaporation). Ces termes d'interaction entre phases sont obtenus par sommation dans chaque maille des échanges entre les gouttes ayant traversé la maille considérée et le gaz. Ils constituent des termes sources à inclure dans les équations eulériennes de conservation de la masse, de l'énergie, de la quantité de mouvement et de la turbulence [7]. Le couplage entre les phases liquide et gazeuse est alors simulé par des calculs successifs de la phase gazeuse en eulérien (en prenant en compte des termes sources diphasiques constants) et de la phase liquide dispersée en lagrangien (en supposant le champ aérodynamique de l'écoulement porteur figé). Le processus est alors répété jusqu'à convergence de l'ensemble, caractérisée par des champs aérothermochimiques et des termes sources de couplage entre phases stationnaires.

\subsection{Conditions aux limites d'un calcul diphasique}

Une des difficultés inhérentes à un calcul lagrangien est la définition de conditions initiales précises pour la trajectographie. En particulier, on doit fixer pour chaque goutte sa position, sa taille, sa vitesse et sa température initiales. Si la température du liquide à l'injection est relativement bien connue, il n'en est pas de même pour la vitesse, et à un degré moindre pour la position et le diamètre.

En effet, les gouttes issues d'un injecteur industriel résultent de la fragmentation primaire d'une nappe ou d'un jet liquide qui libère un nombre important de gouttes de diamètres différents (de 0 à $200 \mu \mathrm{m}$ typiquement), après une distance de cassure de la nappe ou du jet liquide. On comprend donc toute la difficulté pour définir les conditions initiales $(\mathrm{CI})$ des nombreuses gouttes que l'on aura à simuler. Des études paramétriques réalisées à Snecma ont montré que ces conditions initiales conditionnent fortement le résultat final d'un calcul aérothermochimique. 
Plusieurs démarches sont envisageables a priori :

- prévision théorique. L'état de l'art actuel nous montre cependant qu'en général seule une taille moyenne caractéristique du spray peut être prédite par une étude des instabilités d'une nappe liquide alors que nous sommes en présence de polydispersion. Ce sujet constitue donc une voie de recherche importante à l'heure actuelle.

- recours à la mesure. Cette approche nécessite toutefois des mesures extrêmement précises et simultanées de la vitesse, de la taille, et de la fréquence d'injection des gouttes en un point donné. Les mesures PDPA répondent à cette attente mais elles sont réalisées à froid (sans combustion), à pression ambiante, à l'air libre (sans confinement), et à une certaine distance de l'injecteur, ce qui limite leur utilisation pratique.

- pragmatisme et empirisme. On se base sur les essais disponibles qui donnent la forme globale du spray (cône creux) et sur des calculs élémentaires pour fixer les caractéristiques initiales des gouttes. On s'oriente actuellement vers une solution intermédiaire où l'on calcule finement le système d'injection seul à l'aide d'un code $2 \mathrm{D}$ axisymétrique et on impose les CI du brouillard dans un calcul 3D à partir des résultats obtenus par le calcul 2D.

\section{- 5.5 Axes de recherche à développer}

De nombreux problèmes restent à résoudre lorsque l'on utilise une méthode diphasique lagrangienne :

1) la détermination des conditions initiales des gouttes de carburant (taille, position, vitesse). Ainsi, que nous l'avons souligné, ce point est primordial car la qualité des résultats obtenus en dépend fortement. Les recherches sur l'atomisation primaire doivent donc être encouragées même si les résultats ne sont pas envisageables à court terme.

2) l'évolution des caractéristiques du spray en fonction des conditions en pression et température de la chambre de combustion.

3) l'interaction goutte/paroi : la création d'un film liquide par des gouttes impactant une paroi (ruissellement) peut modifier la granulométrie globale du spray et par là même certaines des performances du foyer.

4) l'interaction entre gouttes (coalescence, modification de l'évaporation et de la traînée). Cela suppose que l'on suit simultanément l'évolution d'un nombre très important de gouttes, ce qui rend délicate la prise en compte de ces effets.

5) la validation : une méthode lagrangienne fournit une grande quantité d'informations sur chaque trajectoire. Cela implique le recours à une expérimentation très précise (locale et non intrusive) pour la validation, et cela en milieu éventuellement réactif.

6) pulvérisation secondaire.

\section{VI $\square$ CONCLUSION}

La mise au point de systèmes d'injection représente une phase cruciale lors du développement d'un nouveau foyer aéronautique. En effet, la qualité de l'injection conditionne ou influence la quasi-totalité des performances requises pour un foyer : stabilité, rallumage en altitude, pollution, sollicitation thermique des parois, profil de température en sortie foyer.

Devant la complexité des phénomènes physiques mis en jeu (fragmentation d'une nappe ou d'un jet de carburant, pulvérisation secondaire, ruissellement, dispersion turbulente,...), Snecma mise depuis quelques années sur la modélisation numérique afin de limiter le recours à un empirisme systématique, tout en se basant sur l'expérience capitalisée. Les mesures et la caractérisation expérimentale de plus en plus précises, demeurent toutefois plus que jamais utiles comme éléments de validation de la modélisation et comme analyse fine du dispositif technologique. Les axes de recherche, tant théoriques qu'expérimentaux, restent donc nombreux dans le domaine de la modélisation de la combustion multiphasique dans les moteurs aéronautiques.

\section{BIBLIOGRAPHIE}

[1] S. Meunier, D. ANSART, P. Ciccia, Les foyers de turboréacteurs à géométrie variable : une réponse à la mâtrise de la pollution dans des cycles à haute température, haute pression; AGARD 81 st symposium 1993 Fiuggi.

[2] C. Dejeu, J.L. Schultz, S. Meunier, Calculs aérothermochimiques dans les foyers de réchauffe; AGARD 81st symposium 1993 Fiuggi.

[3] A. PAGE, M. BUFFAT, A parallel unstructured solver for 3D turbulent reacting flows: laminar and turbulent flows, 10th international conference, editions C.Taylor \& J.T Cross, Pineridge Press, Swansea, 1997.

[4] R. GATIGNOL, The faxen formulae for a rigid particle in an unsteady non uniform Stokes flow; Journal Méca Théo et Appl, Vol 1, pp 143-160. 1983.

[5] P. BÊARD, Modélisation lagrangienne de la dispersion et de l'évaporation de gouttes dans un écoulement turbulent instationnaire; Thèse ENSAE décembre 1994

[6] A.K. TOLPADI, A numerical study of two phase flow in gas turbine combustors. AIAA 92-3468, 6-8 july 1992

[7] M.S.Grancher, Simulation de l'évaporation de gouttelettes en écoulement turbulent suivant une approche lagrangienne; Thèse Univ. Rouen juin 1990. 